\section{Comments}

Socioeconomic category influenced the use of the Glasgow Emergency Medical Service, with increased contact rates by children and adults from deprived areas. For elderly people, the highest contact rate was among the most affluent. While the effect of affluence on raising expectations and service use has been reported, this association has not previously been linked to age. ${ }^{5}$

After contacting the emergency service, adults and elderly people from deprived areas were most likely to receive a home visit. The reasons for this are unclear and require further investigation. The lack of association between socioeconomic category and centre visit rates may be attributable to the provision of patient transport for anyone asked to attend a centre: those from deprived areas were far more likely to use the service. The cost of this service for 1998-9 will be around $£ 240000$, yet may still be cheaper than taxi fares or home visits. While this requires economic evaluation, the evidence suggests that a free and accessible patient transport service may contribute to equity of access to out of hours primary care across the socioeconomic spectrum.

We thank Dr James O'Neil, John Easthope, and the staff of the Glasgow Emergency Medical Service for their cooperation; staff of the Robertson Centre for Biostatistics for data entry; Depart- ment of Health Information, Greater Glasgow Health Board for routine data; Ms Michere Beaumont for secretarial support Professor Graham Watt for his discussions. Ethical approval was obtained from the Greater Glasgow Community and Primary Care Research Ethics Committee. The Public Health Research Unit is supported by the Chief Scientist Office of the Scottish Office Department of Health, but the views expressed in this paper are those of the authors alone.

Contributors: COD contributed to the conception and design of the study, collection and analyses of the data, and coordinated writing of the paper; she will act as guarantor for the paper. AMcC performed statistical analyses and contributed to writing the paper. KM, ND, and PW contributed to the conception and design of the study, collection of the data, and to writing the paper. SJR contributed to the conception and design of the study, collection and analyses of the data, and to writing the paper.

Funding: Glasgow Emergency Medical Service contributed towards data entry costs.

Conflict of interest: None.

1 Payne F, Jessopp L, Dale J. Second national survey of GP co-operatives: report. London: Department of General Practice and Primary Care, King's College School of Medicine and Dentistry, 1997.

2 Hallam L. Out of hours primary care. BMJ 1997;314:157-8.

3 Carlisle R, Groom LM, Avery AJ, Boot D, Earwicker S. Relation of out of hours activity by general practice and accident and emergency services with deprivation in Nottingham: longitudinal survey. BMJ 1998;316: $520-3$.

4 Carstairs V, Morris R. Deprivation and health in Scotland. Aberdeen: Aberdeen University Press, 1991

5 Pitts J, Whitby M. Out of hours workload of a suburban general practice: deprivation or expectation. BMJ 1990;300:1113-5.

(Accepted 23 October 1998)

\title{
Trend analysis of socioeconomic differentials in deaths from injury in childhood in Scotland, 1981-95
}

\author{
Anita Morrison, David H Stone, Adam Redpath, Harry Campbell, John Norrie
}

Injuries are a leading cause of death and disability among children in the United Kingdom, costing an estimated $£ 200$ million annually in direct costs to the NHS. ${ }^{1}$ Recent research from England and Wales suggests that death rates from injury and poisoning have fallen in both sexes and all social classes. ${ }^{2}$ The decline in those of lower social class has been much smaller, however, resulting in widening socioeconomic differentials in mortality from injury. To determine whether this phenomenon has also occurred in Scotland we examined time trends in mortality from injury in Scottish children for 1981-95.

\section{Subjects, methods, and results}

National data on external cause of death (injury and poisoning; E800-999 of the ninth edition of the International Classification of Diseases) for children aged 0-14 years were obtained from the registrar general for Scotland for the period 1981-95. Socioeconomic status was measured with Carstairs' deprivation index on the basis of characteristics of Scottish postcode sectors. This involves a continuous scale of $1-7$, where 1 is most affluent and 7 is most deprived. The per cent reduction in fatalities from 1981 to 1995 was estimated with Poisson regression models for deprivation categories 1-2, 3-5, and 6-7 and for all deprivation categories combined. In all models a log linear time trend was found to be adequate. Confidence intervals were adjusted for overdispersion. ${ }^{4}$ A further Poisson regression model compared the least and most deprived groups.

In $1981-95,1728$ Scottish children ( $65 \%$ boys) aged $0-14$ years died as a result of an injury or poisoning. Overall, there was a decline of 58\% (95\% confidence interval $45 \%$ to $68 \%$ ) from 1981 to 1995 with observed death rates per 100000 averaging 13.2 for 1981-93 compared with an average of 7.4 from 1993-5. The proportion of deaths due to injury decreased only slightly, from $14 \%$ of deaths in children in 1981-3 to $12 \%$ in $1993-5$.

Throughout the study period children residing in areas of relatively greater deprivation (6-7) experienced higher mortality from injury than children living in more affluent areas (1-2 and 3-5) (figure). Similar proportional decreases were observed in all deprivation categories over the study period. Between 1981 and 1995 mortality from injury decreased by $60 \%$ for categories $1-2$ and 3-5 (27\% to $78 \%$ and $47 \%$ to $70 \%$, respectively) and by $53 \%$ (27\% to $70 \%)$ for categories 6-7. The observed average mortality per 100000 for 1981-3 compared with $1993-5$ was 9.9 versus 5.6, 13.3 versus 7.0 , and 17.0 versus 10.7 for categories $1-2,3-5$, and 6-7, respectively. The risk ratio between the most and least deprived groups was 2.29 (1.82 to 2.88).
Paediatric

Epidemiology and Community Health (PEACH) Unit, Department of Child Health, University of Glasgow, Glasgow G3 8SJ Anita Morrison, research officer David H Stone, director

Information and Statistics Division, Scottish Health Service, Trinity Park House, Edinburgh EH5 3SQ

Adam Redpath, principal statistician

continued over

BMJ 1999;318:567-8 
Department of Public Health Sciences, Medical School, University of Edinburgh, Edinburgh EH8 9AG

Harry Campbell, senior lecturer in epidemiology

Robertson Centre for Biostatistics, Boyd Orr Building,

University of Glasgow, Glasgow G12 8QQ John Norrie, senior statistician

Correspondence to: Ms Morrison Anita.Morrison@ clinmed.gla.ac.uk

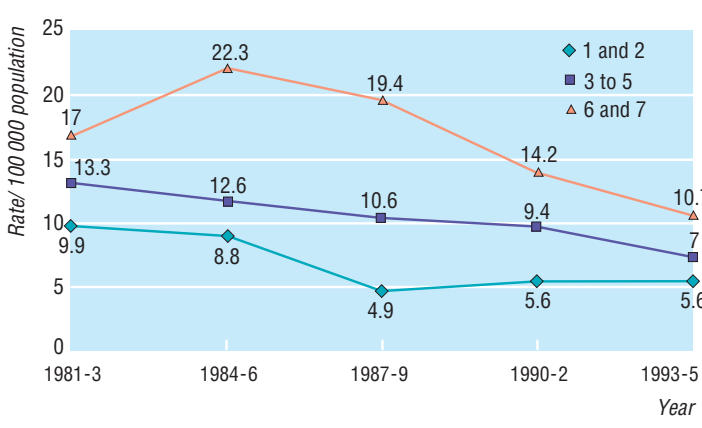

Mortality from injury per 100000 children by category of deprivation, 1981-95

There was no evidence that this had changed over the study period $(\mathrm{P}=0.56)$.

\section{Conclusions}

Despite overall improvements in childhood mortality from injury in England and Wales, children in the lower socioeconomic groups seem to be experiencing progressively increasing relative risks of injury in comparison with those in higher socioeconomic groups, ${ }^{2}$ possibly as a result of a differential impact of health education efforts. Our data suggest that this may not be the case in Scotland. The reason for this difference north and south of the border is unclear. One hypothesis is that, unlike in England and Wales, measures to prevent injury in children are exerting an equal effect on all socioeconomic groups in Scotland. An alternative explanation is that the decline has occurred independently of specific preventive efforts.

We cannot rule out the possibility that the cross border differences are due to the way socioeconomic status is measured. The Carstairs' deprivation scores adopted in Scotland and the social class definitions used in the analysis of England and Wales data are not necessarily directly comparable. The Carstairs system adopts a measure of "area" deprivation, whereas the measure of social class used in England and Wales categorises the population into five classes based on individual occupational status. This may be important as there is some evidence that the characteristics of communities, as well as those of individuals, exert an impact on the health of the population. ${ }^{5}$ Further investigation of the apparently divergent socioeconomic patterns of mortality from injury in children within the United Kingdom is necessary.

Contributors: AM discussed core ideas, participated in data analysis, data interpretation, and writing of the paper. DS contributed to the initiation of the study, discussed core ideas, participated in data analysis and interpretation, and contributed to the writing of the paper. AR contributed to the initiation of the study, provided the data, participated in data analysis and interpretation, and edited the paper. HC contributed to the initiation of the study, participated in data interpretation, and edited the paper. JN provided statistical advice. DHS is guarantor for the study.

Funding: None.

Competing interests: None declared.

1 Jarvis S, Towner E, Walsh S. Accidents. In: Botting B, ed. The health of our children. Decennial supplement. London: HMSO, 1995.

2 Roberts I, Power C. Does the decline in child injury mortality vary by social class? A comparison of class specific mortality in 1981 and 1991. BMJ 1996;313:784-6

3 Carstairs V. Deprivation and health in Scotland. Aberdeen: Aberdeen University Press, 1991.

4 Lindsey JK. Modelling frequency and count data. Oxford: Clarendon Press, 1995.

5 Davey Smith G, Ben-Shlomo Y. Inequalities in health: what is happening and what can be done. In: Scally G, ed. Progress in public health. London: Financial Times Health Care, 1997.

(Accepted 15 September 1998)

\section{Routine screening of children returning home from the tropics: retrospective study}

Marianne L Brouwer, Jules J M Tolboom, Jaap H J Hardeman

Department of

Paediatrics

University Hospital

Nijmegen

St Radboud,

PO Box 9101,

6500 HB Nijmegen,

Netherlands

Marianne L

Brouwer,

medical student

Jules J M Tolboom

senior consultant

Haskoning BV

Consultants,

PO Box 151,

6500 AD Nijmegen

Jaap H J Hardeman,

medical adviser

Correspondence to:

Dr Tolboom

j.tolboom@ckskg.

azn.nl

BMJ 1999;318:568-9
Parents who move to the tropics with their children often worry about tropical diseases. Most of the data available on screening children returning home from living in the tropics are from 1952-63 from American children. ${ }^{1}$ More recent findings in asymptomatic people returning to the United Kingdom have been reported. ${ }^{2}$ We aimed to determine whether it is worthwhile to routinely screen children without symptoms returning home from the tropics.

\section{Subjects, methods, and results}

During 1987-95 all children visiting our hospital for a medical check up after a stay in the tropics were studied. This check up was obligatory under their parents' terms of employment. Our protocol included a questionnaire (completed by the parents), physical examination of the child, and additional investigations. These investigations included a full blood cell count; eosinophil count; liver function tests; urea and creatinine concentrations; hepatitis A and B serology; thick smear; schistosoma and filaria serology (if the child had stayed in endemic areas); urine analysis; stool culture; analysis of three stool samples for ova and cysts; tuberculin skin test; and a chest $x$ ray if indicated. The same specialist (IJMT) performed all consultations. Data were analysed with EpiInfo version 5.0.

\section{Results}

A total of 216 children (103 girls) were seen during 282 check ups (some children had lived in the tropics more than once) (table). In 175 (62\%) cases (check ups), children had lived in sub-Saharan Africa. Ninety three children $(43 \%)$ were born in the tropics. The length of stay overseas ranged from 3 months to 13 years; in 150 $(53 \%)$ cases the stay was 1-3 years. Age at first check up ranged from 3 months to 16 years (mean 4.75 , median 